\title{
Growth of N-benzyl-2-methyl-4-nitroaniline (BNA) single crystal fibers by micro -pulling down method
}

*Kei Kamada ${ }^{1,2}$, Yuma Takida ${ }^{3}$, Hiroaki Minamide ${ }^{3}$, Yasuhiro Shoji ${ }^{2,4}$, Shunsuke Kurosawa $^{1,4}$, Yuui Yokota ${ }^{1}$, Yuji Ohashi $^{4}$, Akira Yoshikawa ${ }^{1,2,4}$

\section{${ }^{1}$ Tohoku University}

New Industry Creation Hatchery Center, 6-6-10 Aoba, Aramaki, Aoba-ku, Sendai, Miyagi,

$$
\begin{aligned}
& \text { 980-8579, Japan } \\
& { }^{2} \text { C\&A corporation }
\end{aligned}
$$

T-Biz, 6-6-10 Aoba, Aramaki, Aoba-ku, Sendai, Miyagi 980-8579, Japan

${ }^{3}$ Tera-Photonics Research Team, RIKEN Center for Advanced Photonics, RIKEN 519-1399, Aramaki-aoba, Aoba-ku, Sendai, Miyagi 980-0845, Japan

${ }^{4}$ Tohoku University

Institute for Materials Reseach, 2-1-1 Katahira Aoba-ku, Sendai, Miyagi, 980-8577, Japan 


\begin{abstract}
N-benzyl-2-methyl-4-nitroaniline (BNA) fiber single crystals were grown by the micro-pulling down $(\mu-P D)$ method. Crucible material and its shape were selected based on wetting of the crucible material by liquid BNA. As a result, BNA fiber single crystal with about 1-2 mm diameter was successfully grown. Powder XRD analysis and x-ray rocking curve measurements were performed. Widely-tunable THz-wave generation from $4 \mathrm{THz}$ to $14 \mathrm{THz}$ was demonstrated using the BNA fiber crystal grown by $\mu$-PD method.
\end{abstract}

Keywords: A1. Directional solidification, A2. Single crystal growth, B1. Organic compounds, B2. Nonlinear optic materials 


\section{Introduction}

Terahertz (THz) technology has recently undergone considerable development [1], and $\mathrm{THz}$ radiation has attracted great attention from a growing number of applications, including biomedical imaging, security, medicine, art conservation, and nondestructive testing [2-6]. A monochromatic THz-wave sources [7-10] using a nonlinear optical (NLO) process were employed in developing of number of such applications, but the advancement of the broadband sources and the sources that possess some degree of frequency agility is desirable. Recently an ultra-broadband capability using difference-frequency generation (DFG) was demonstrated using organic crystals of 4-dimethylamino-N-methyl-4-stilbavolium tosylate (DAST) [11-15] and N-benzyl-2-methyl-4-nitroaniline (BNA) [16-20]. The tuning ranges of monochromatic THz-wave sources based on organic materials were much wider than those of inorganic materials. BNA, which was invented by Hashimoto et al. [17-19], is a promising material for wideband, efficient, and high-power THz-wave generation because of its large second-order optical nonlinearity. The BNA-DFG monochromatic THz-wave was tunable over the range $0.1-15 \mathrm{THz}$.

Development of crystal growth technology that is suitable for fabrication of high-quality crystals with preferable orientation is important for $\mathrm{THz}$ generation with organic crystals. In general, the molecular mass of organic materials is relatively large and their structure is complex as compared with inorganic materials. In addition, binding forces between organic molecules such as the Van der Waals force and hydrogen bonding are relatively weak. Therefore, it is generally difficult to grow high-quality large-size organic single crystals. Thus, optimization of the growth conditions for such crystals is still an important challenge.

Single-crystalline BNA was first obtained from the melt phase by the Bridgman method. Its crystal structure and basic physical properties have been examined in [19,21,22]. Although the Bridgman method is well-established technique in the field of inorganic crystals growth, it may be not suitable for crystal growth of organic materials demonstrating high crystallinity. This is because the crystals grown by the Bridgman method tend to have lattice defects and distortions originated from mechanical and thermal stresses induced from the crucible. Alternatively, Notake et al. reported solution growth of BNA. BNA crystal plates of better crystallinity could be grown from ethanol solution. Thus, solution grown BNA crystals had rocking curve width of 30 arcseconds that was three times narrower than that of crystals produced by the Bridgman method [23].

The micro-pulling down ( $\mu$-PD) technique is a melt crystal growth method that was intensively developed since 1992. In this method, the melt residing in a crucible is transported in downward direction 
through the microcapillary channel(s) positioned in the bottom of the crucible. Thus, the solidification of the melt is occurred just below the crucible bottom on the crystal-melt interface. Appropriate configuration of a die at the crucible bottom and properly selected temperature gradient allow control of the crystal shape and its orientation. The crystal cross-section is ranged from 0.1 to $10 \mathrm{~mm}$. In addition, this method does not demonstrate considerable effect of the crucible on formation of mechanical and/or thermal stresses because the crystal is not in direct physical contact with the crucible [24,25]. In this report, BNA fiber single crystals were grown by the $\mu$-PD method. Crucible materials and crucible shape were selected considering wetting of the crucible materials by BNA melt. X-ray rocking curve measurements were performed to evaluate crystallinity of as produced fibers.

\section{Experimental}

BNA single crystals were grown using a $\mu$-PD growth system that was basically analogous to the $\mu-P D$ arrangements that have been described in the earlier reports (see e.g., detailed descriptions in Refs. [24,25]). To establish appropriate growth atmosphere, the growth chamber was firstly evacuated with a rotary pimp and then filled with Ar gas. A resistive heater with a thermocouple controlled temperature of a crucible. The temperature gradient below the crucible was regulated by cooling water from a thermostatic bath and temperature below the crucible was monitored using another thermocouple.

Before beginning the crystal growth experiments, the wetting angle between BNA melt and various crucible materials were examined using the same $\mu$-PD growth apparatus. BNA raw material (Furukawa co. 1td.) was melted on the plates made of SUS304 stainless steel, aluminum, copper, Pyrex glass, polypropylene, and nylon in Ar atmosphere. Wet angles were inspected with a CCD camera. Fig. 1 demonstrates results of the wetting angles observations. Wetting angles of SUS304, aluminum and polypropylene were 39,37 , and 40 degree, respectively. These substances were considered as suitable ones for application as crucible materials according to previous reports [24,25]. Therefore, SUS304 and polypropylene crucibles with $2 \mathrm{~mm}$ diameter die and $0.5 \mathrm{~mm}$ diameter capillary outlets were designed and produced.

For the actual crystal growths, typical pulling rates applied were $0.05-0.07 \mathrm{~mm} / \mathrm{min}$ and the diameter of as produced solids were around $2 \mathrm{~mm}$. The crystals were grown from the SUS304 and polypropylene crucibles under the Ar atmosphere. A capillary glass tubes with inner channel of $0.2 \mathrm{~mm}$ in diameter were used as a seed. After the growths, the fragments of the grown crystals were crushed and ground into a powders in a mortar. The thermal characteristics of the BNA raw material was analysed by thermogravimetric (TG) and differential thermal (DTA) analyses at a heating rate of $2{ }^{\circ} \mathrm{C} / \mathrm{min}$ and a cooling rate of $0.25^{\circ} \mathrm{C} / \mathrm{min}$ under Ar gas at a flow rate of $300 \mathrm{ml} / \mathrm{min}$. Powder X-ray diffraction analysis 
was carried out in the $2 \theta$ range of $5-60^{\circ}$ using the RINT Ultima (RIGAKU) diffractometer. For these measurements, the $\mathrm{CuK} \alpha \mathrm{X}$-ray source was used, and the accelerating voltage and current were $40 \mathrm{kV}$ and $40 \mathrm{~mA}$, respectively. Rocking curve measurements were performed by Rigaku-ATX.

\section{$3 \quad$ Results and discussion}

The results of the TG/DTA analysis were used to measure melting and solidification points of the BNA raw material. These temperatures were found to be 106 and $65^{\circ} \mathrm{C}$, respectively. These results were used to control temperature of the resistive heater and the cooling water.

The BNA crystals grown from the SUS304 and polypropylene crucibles are illustrated in Fig. 2. In the case of growth from the SUS304 crucible, the crystal diameter could not be controlled. The as grown crystal was not transparent and had cracks. In contrast, a transparent and crack-free BNA crystal of 1-2 $\mathrm{mm}$ in diameter and $12 \mathrm{~mm}$ in length was grown from the polypropylene crucible. In the case of BNA growth using the SUS304 crucible, melt/solid interface was not stable and the melt easily got up the die. Thermal conductivity of SUS304 and polypropylene are 16.7 and $0.12 \mathrm{~W} /(\mathrm{m} \cdot \mathrm{K})$, respectively. In the case of the SUS304 crucible, the melt lost its latent heat and crystallized more rapidly because of its higher thermal conductivity. It was assumed that this rapid crystallization caused instability of the melt/solid interface.

Powder X-ray diffraction (XRD) analysis was performed to identify structure of the grown BNA crystal. The results of the powder XRD of the grown BNA crystal produced from the polypropylene crucible are demonstrated in Fig. 3. All the obtained peak were identified as those typical for the Orthorhombic Pna21 (33) BNA phase. The lattice constants were calculated to be 7.42(a), 21.46(b), and $8.13(\mathrm{c})$. These results were in good agreement with those reported in the past $[19,21]$

Rocking curve measurements were performed by Rigaku-ATX at $2 \theta=8.231$ of $<020>$ peak. Slit size was set to be $0.5 \mathrm{~mm} \times 0.5 \mathrm{~mm}$. CuKa X-rays generated at $40 \mathrm{kV}$ and $30 \mathrm{~mA}$ were irradiated to the [010] face of the BNA crystal grown by the $\mu$-PD method. The results were compared with those obtained in identical conditions using BNA grown from the solution using the same starting material (Fig. 4). The samples produced by $\mu$-PD growth and a solution grown had the line widths of rocking curves of 19.7 and 63.4 arcsec, respectively. Thus, the $\mu$-PD grown BNA crystal demonstrated superior crystallinity as compared to those of the reported BNA crystals grown by the solution growth ( $\sim 30$ arcsec) and Bridgman method ( 100 arcsec) [23].

In order to confirm the widely-tunable THz-wave radiation from the $\mu$-PD grown BNA crystal, DFG experiment was conducted. The DFG system used in this study was essentially identical to the experimental setup described in $[16,20]$. Dual-wavelength output from dual-KTP optical parametric 
oscillator (OPO) was used to pump the as-grown BNA crystal. One of the pump wavelengths was fixed at $910 \mathrm{~nm}$, and the other one was controlled to tune the THz-wave frequency up to $20 \mathrm{THz}$. The total pulse energy of pump waves and repetition rate were $0.2 \mathrm{~mJ} /$ pulse and $100 \mathrm{~Hz}$, respectively. The pump waves were focused on the BNA crystal by a lens with the focal length of $200 \mathrm{~mm}$. The THz-wave radiation via DFG was detected by a $4 \mathrm{~K}$ Si bolometer. Figure 5 shows the THz-wave output spectrum from the $\mu$-PD grown BNA crystal. Widely-tunable THz-wave generation from $4 \mathrm{THz}$ to $14 \mathrm{THz}$ was successfully established from the $\mu$-PD grown BNA fiber crystal.

\section{Conclusion}

BNA fiber single crystals were grown by the $\mu$-PD method. Crucible material and its shape were optimized according to wetting measurements between the BNA and candidate crucible materials. Finally, the BNA fiber single crystal of about 1-2 mm in diameter was successfully produced using the polystyrene crucible. The as grown BNA crystal was single-phase material with orthorhombic structure. The line width of the rocking curve of the BNA crystal was 19.7 arcsec. This value is superior to those of reported BNA crystals grown by the solution growth ( $\sim 30 \operatorname{arcsec})$ and Bridgeman method ( 100 arcsec). THz-wave generation from $4 \mathrm{THz}$ to $14 \mathrm{THz}$ was demonstrated on the $\mu$-PD grown BNA fiber. This way, advantages of the $\mu$-PD growth of the BNA crystals was experimentally verified. 


\section{Acknowledgement}

This work was partially supported by Development of Systems and Technology for Advanced Measurement and Analysis, Japan Science and Technology Agency (JST), Adaptable \& Seamless Technology Transfer Program through Target-driven R\&D (A-STEP), the Health Labor Sciences Research Grant, The Ministry of Health Labor and Welfare and Czech MEYS KONTAKT Cz-jp collaboration LH14266 project, Crystal Clear Collaboration in CERN, and EC Marie Curie Initial Training Network LUMINET, no. 316906. In addition, we would like to thank following persons for their support: Mr. Takanori Endo, Mr. Akira Harako, Ms. Chisato Suzuki and Yoshiyuki Usuki (Furukawa co. ltd.).

\section{References}

[1]. R. Sanchez, and X. C. Zhang, IEEE J. Sel. Top. Quantum Electron. 14(2), (2008) 260

[2]. T. Dekorsky, V. A. Yakovlev, W. Seidel, M. Helm, and F. Keilman, Phys. Rev. Lett. 90, (2003) 05508 .

[3]. H. Yoneyama, M. Yamashita, S. Kasai, K. Kawase, H. Ito, and T. Ouchi, Opt. Commun. 281(7), (2008) 1909.

[4]. K. Kawase, Y. Ogawa, Y. Watanabe, and H. Inoue, Opt. Exp., 11, (2003) 2549

[5]. K. Fukunaga, Y. Ogawa, S. Hayashi, and I. Hosako, IEICE Electron. Express 4(8), (2007) 258.

[6]. S. Ohno, A. Hamano, K. Miyamoto, C. Suzuki, and H. Ito, J. Eur. Opt. Soc. Rapid Publ. 4, (2009) 09012.

[7]. K. Kawase, M. Sato, T. Taniuchi, and H. Ito, Appl. Phys. Lett. 68(18), (1996) 2483.

[8]. G. D. Boyd, T. J. Bridges, C. K. N. Patel, and E. Buehler, Appl. Phys. Lett. 21(11), (1972) 553.

[9]. W. Shi, Y. J. Ding, N. Fernelius, and K. Vodopyanov, Opt. Lett. 27(16), (2002) 1454

[10]. T. Tanabe, K. Suto, J. Nishizawa, K. Saito, and T. Kimura, Appl. Phys. Lett. 83(2), (2003) 237.

[11]. K. Kawase, M. Mizuno, S. Sohma, H. Takahashi, T. Taniuchi, Y. Urata, S. Wada, H. Tashiro, and H. Ito, Opt. Lett. 24(15), (1999) 1065.

[12]. K. Kawase, T. Hatanaka, H. Takahashi, K. Nakamura, T. Taniuchi, and H. Ito, Opt. Lett. 25(23), (2000) 1714.

[13]. T. Taniuchi, J. Shikata, and H. Ito, Electron. Lett. 36(16), (2000) 1414.

[14]. H. Ito, K. Suizu, T. Yamashita, A. Nawahara, and T. Sato, Jpn. J. Appl. Phys. 46(11), (2007) 7321.

[15]. K. Suizu, K. Miyamoto, T. Yamashita, and H. Ito, Opt. Lett. 32(19), (2007) 2885.

[16]. K. Miyamoto, H. Minamide, M. Fujiwara, H. Hashimoto, and H. Ito, Opt. Lett. 33(3), (2008) 252.

[17]. H. Hashimoto, Y. Okada, H. Fujimura, M. Morioka, O. Sugihara, N. Okamoto, and R. Matsushima, Jpn. J. Appl. Phys. 36(Part 1, No. 11), (1997) 6754.

[18]. H. Hashimoto, H. Takahashi, T. Yamada, K. Kuroyanagi, and T. Kobayashi, J. Phys. Condens. 
Matter 13(23), (2001) L529.

[19]. M. Fujiwara, K. Yanagi, M. Maruyama, M. Sugisaki, K. Kuroyanagi, H. Takahashi, S. Aoshima, Y. Tsuchiya, A. Gall, and H. Hashimoto, Jpn. J. Appl. Phys. 45(11), (2006) 8676.

[20]. K. Miyamoto, S. Ohno, M. Fujiwara, H. Minamide , H.Hashimoto, and H. Ito, Optics Express 17(17), (2009) 14832

[21]. K. Kuroyanagi, M. Fujiwara, H. Hashimoto, H. Takahashi, S. Aoshima, and Y. Tsuchiya, Jpn. J. Appl. Phys. 45(29), (2006) L761.

[22]. M. Fujiwara, M. Maruyama, M. Sugisaki, H. Takahashi, S. Aoshima, R. J. Cogdell, and H. Hashimoto, Jpn. J. Appl. Phys. 46(4A), (2007) 1528.

[23].T. Notake, K. Nawata, H. Kawamata, T. Matsukawa, and H. Minamide, Opt. Meter. Express 2(2), (2012) 119.

[24]. T. Fukuda, V.I. Chani, Shaped crystals: micro-pulling-down technique and growth, Springer, Berlin, New York, 2007.

[25]. A. Yoshikawa, B. M. Epelbaum, K. Hasegawa, S. D. Durbin and T. Fukuda J. Cryst. Growth 205 (1999) 305. 
Figure captions

Figure 1. Illustration of measurements of wettability (contact angles) between the BNA melt and various candidate crucible materials.

Figure 2. Views of the BNA crystals grown from a) SUS304 and b) polypropylene crucibles.

Figure 3. Powder XRD data for the grown BNA crystal.

Figure 4. Results of the X-ray Rocking curve analysis for the BNA crystals produced by $\mu$-PD and solution grows. $(2 \theta=8.231<020>$, Slit size $: 0.5 \mathrm{~mm} \times 0.5 \mathrm{~mm}$. CuK $\alpha 40 \mathrm{kV}-30 \mathrm{~mA})$.

Figure 5. THz-wave output spectrum of the BNA crystal obtained via DFG. 
Kamada,et al, Figure 1

SUS: $39^{\circ}$

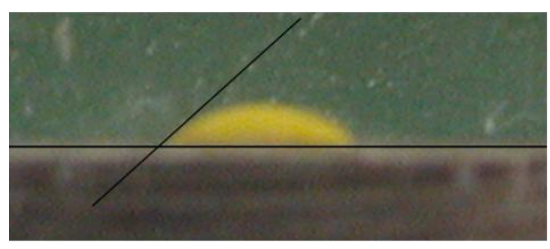

$\mathrm{Al}: 37^{\circ}$

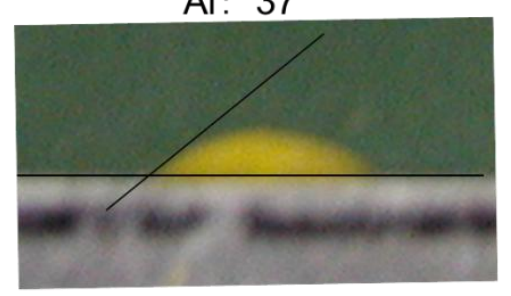

$\mathrm{Cu}: 27^{\circ}$

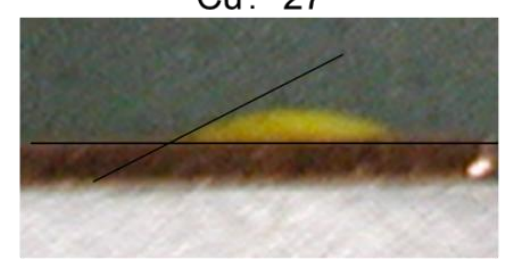

glass: $30^{\circ}$

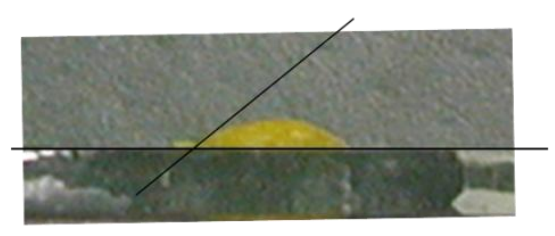

Polypropylene: $40^{\circ}$

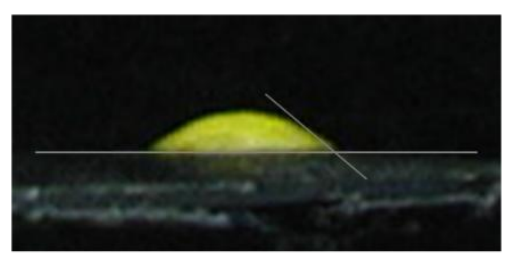

Nylon: $15^{\circ}$ 
a)

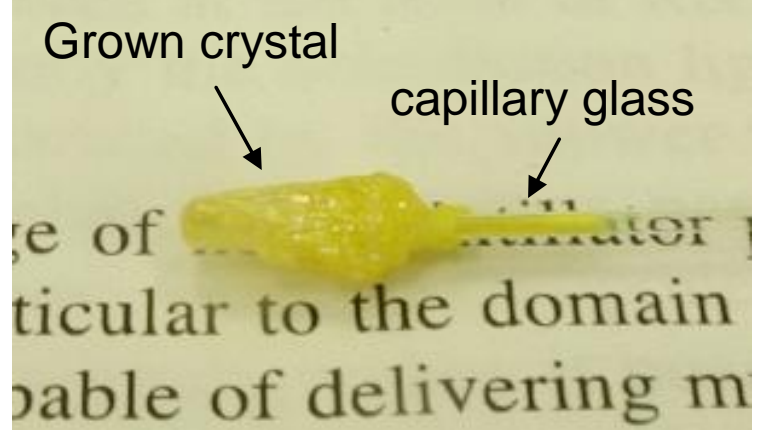

b)

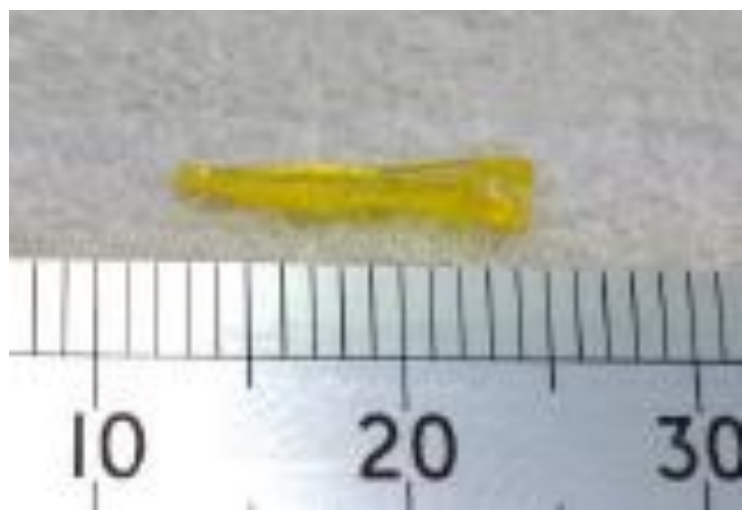


Kamada,et al, Figure 3

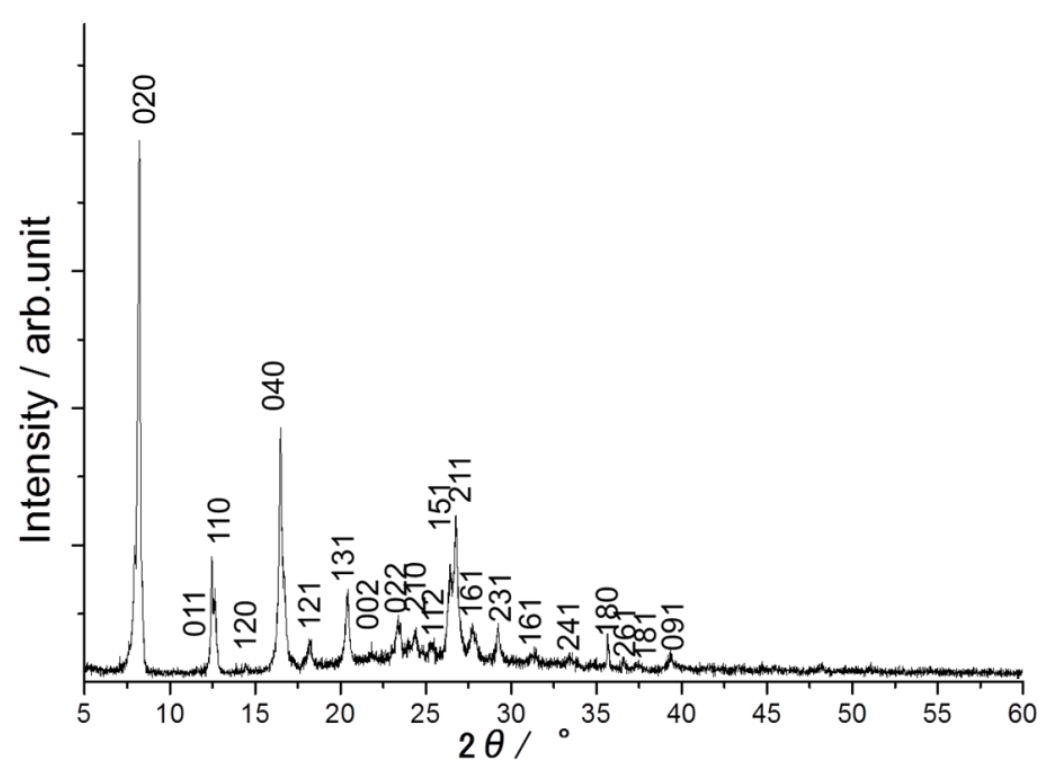


Kamada,et al, Figure 4

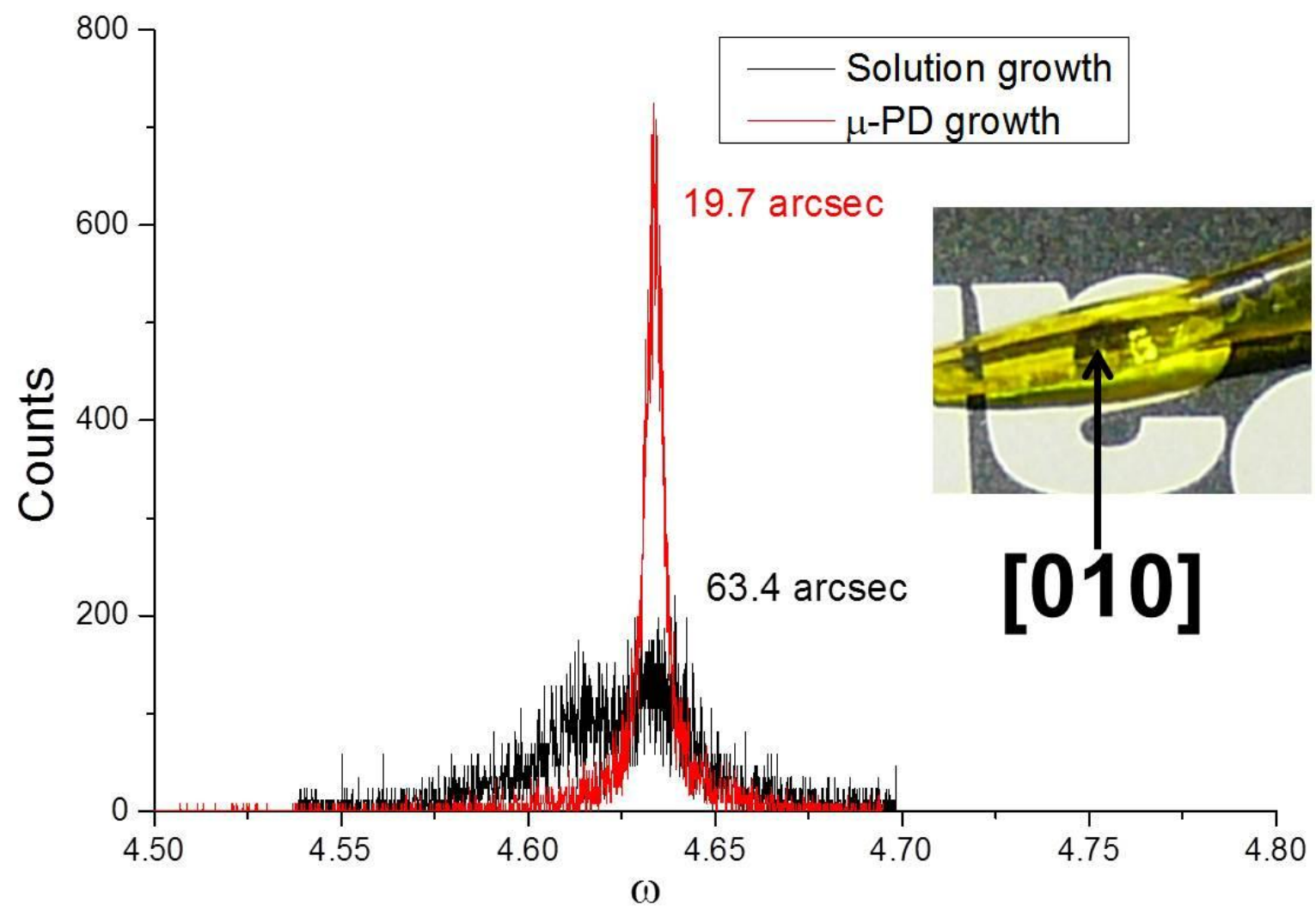


Kamada,et al, Figure 5

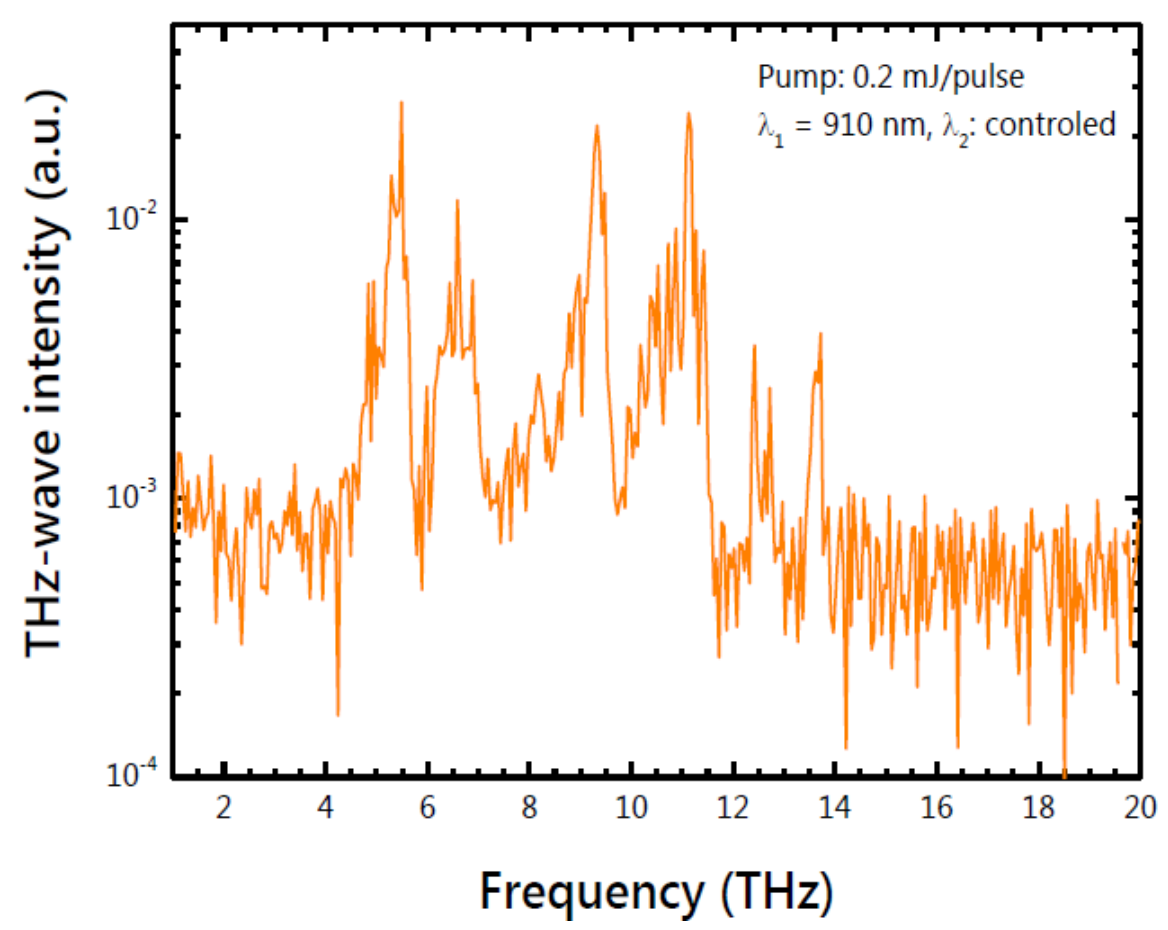

